Review

\title{
Metal Foaming Investigated by X-ray Radioscopy
}

\author{
Francisco Garcia-Moreno $^{1,2, *}$, Manas Mukherjee ${ }^{1,2}$, Catalina Jiménez ${ }^{1,2}$, Alexander Rack ${ }^{3}$ \\ and John Banhart ${ }^{1,2}$
}

1 Helmholtz-Centre Berlin for Materials and Energy, Hahn-Meitner-Platz 1, Berlin 14109, Germany; E-Mails: manas.mukherjee@helmholtz-berlin.de (M.M.); catalina.jimenez@helmholtz-berlin.de (C.J.); banhart@helmholtz-berlin.de (J.B.)

2 Technical University Berlin, Germany, Hardenbergstrasse 36, Berlin 10623, Germany

3 European Synchrotron Radiation Facility, BP 220, Grenoble 38043, France;

E-Mail: alexander.rack@esrf.fr

* Author to whom correspondence should be addressed; E-Mail: garcia-moreno@helmholtz-berlin.de; Tel.: +49-30-8062-42761; Fax: +49-30-8062-3059.

Received: 12 October 2011; in revised form: 21 November 2011 / Accept: 19 December 2011 / Published: 27 December 2011

\begin{abstract}
The use of X-ray radioscopy for in-situ studies of metal foam formation and evolution is reviewed. Selected results demonstrate the power of X-ray radioscopy as diagnostic tool for metal foaming. Qualitative analyses of foam nucleation and evolution, drainage development, issues of thermal contact, mold filling, cell wall rupture and more are given. Additionally, quantitative analyses based on series of images of foam expansion yielding coalescence rates, density distributions, etc., are performed by dedicated software. These techniques help us to understand the foaming behavior of metals and to improve both foaming methods and foam quality.
\end{abstract}

Keywords: metal foam; X-ray; radioscopy; synchrotron radiation

\section{Introduction}

Solid metallic foams are promising materials for a variety of applications [1]. Since their structure is as complex as that of all foams, their study is challenging [2]. To understand metal foam formation, evolution and stabilization we need to study them in-situ in the liquid state before and during 
solidification, as the processes at this stage clearly influence the final solid foam structure and properties.

Radiographic imaging techniques based on photons or neutrons offer the chance to observe the inner structure of the samples [3]. A series of radiographies recorded in real time with an acceptable time resolution is known as radioscopy. In foams, radioscopy allows us to study in-situ different processes like expansion, macroscopic pore structure evolution, density distribution, coalescence events, etc. We will concentrate on X-ray radioscopy, as this method offers the best spatial and temporal resolutions and optimal contrast between the metallic matrix and the gas-containing pores. We will present the evolution of X-ray imaging to monitor metal foaming from the first reported activities in the year 2000 to the latest developments.

\section{X-ray Synchrotron Radioscopy in Material Science}

X-ray radiation provides the opportunity to "see" through otherwise opaque materials such as metals or ceramics. Synchrotron radioscopy featuring good spatial (in the $\mu \mathrm{m}$-range) and time resolution $(<1 \mathrm{~s})$ is suitable for in-situ analyses of various phenomena in materials science and was successfully applied in recent years [4-11] as will be reviewed for a few examples closely related to the foaming process including solidification, diffusion and flow in metals.

\subsection{Solidification of Metals}

The solidification microstructure has a pronounced influence on the mechanical properties of metallic alloys. It can be either columnar, equiaxed or mixed, and the factors influencing its formation and evolution need to be studied. In-situ synchrotron X-ray radioscopy of the solidification process was performed in the past years, for example by groups in Norway and France, which studied the solidification behavior of different alloys [4-8]. They were able to observe directly dendrite fragmentation, porosity formation, directional and equiaxed dendritical solidification, phase separation and even fluctuations in elemental concentration at the solidification front in Al-based alloys. X-ray transparent Bridgman and isothermal furnaces were used.

\subsection{Diffusion in Metals}

Until now, ex-situ and post-mortem analyses could not reveal the kinetics of diffusion. Effects such as convection-driven fluid flow could now be observed. An in-situ X-ray technique for tracking concentration profiles by exploiting Beer-Lambert's law was presented and chemical diffusion measurements in Al-Ni-Ce melts are compared with self-diffusion and thermodynamic data in order to estimate the influence of thermodynamic forces on diffusion [9]. Further X-ray-supported diffusion experiments will be carried out under microgravity conditions in the frame of the XRMON (X-ray monitoring) project conducted with the support of European Space Agency (ESA) and the German Space Agency (DLR). 


\subsection{Flow of Melts}

The flow of the semi-solid aluminum-germanium alloy Al-Ge32 (wt.\%) through a constricted opening into a small cavity was monitored by in-situ radioscopy using white hard X-ray synchrotron radiation [10,11]. The acquisition rate was 67 fps. Liquid drainage and de-/re-agglomeration of small aluminum-rich solid particle clusters - associated with the slurry's thixotropic behavior - could be visualized. Such studies can help in improving the production of both high-quality and light-weight metallic components using semi-solid casting techniques. The information obtained from experimental flow kinetics can be further used as input for simulations, validation of models or optimization of injection molding tools.

\section{X-ray Synchrotron Radioscopy on Metal Foams}

Third-generation synchrotron light sources deliver a polychromatic X-ray photon flux density high enough to perform radiography with micro-resolution in both space and time [12-15]. The rapid development of imaging hardware, especially in the field of CMOS sensors, and their continuously improving sensitivity now allows for extraordinarily high recording speeds, with exposition times down to $1 \mu \mathrm{s}$ for a considerable (at least $10 \times 20 \mathrm{~mm}^{2}$ ) field of view. The optimized use of so-called $\mathrm{X}$-ray inline phase contrast (due to the coherence properties of an X-ray synchrotron beam) allows for the best possible contrast for our cellular material. Additionally, radiation hard but highly sensitive and efficient single crystal scintillators such as LuAG:Ce or YAG:Ce crystals are employed. This permits us to follow the overall process and resolve in-situ not only slow processes ( $>1 \mathrm{~ms}$ exposition time) such as pore nucleation and growth, foam expansion or drainage, or simply observe the overall foaming process, but also very fast ones ( $\leq 1 \mathrm{~ms}$ exposition time) such as cell wall rupture, bubble coalescence, rapid bubble motions or oscillations. Figure 1 shows the improvement of time resolution for X-ray synchrotron radioscopy achieved in the past years as reported in the literature [14-18] and recent results.

Figure 1. Evolution of time resolution for in-situ synchrotron X-ray radioscopy applied to visualize metal foaming.

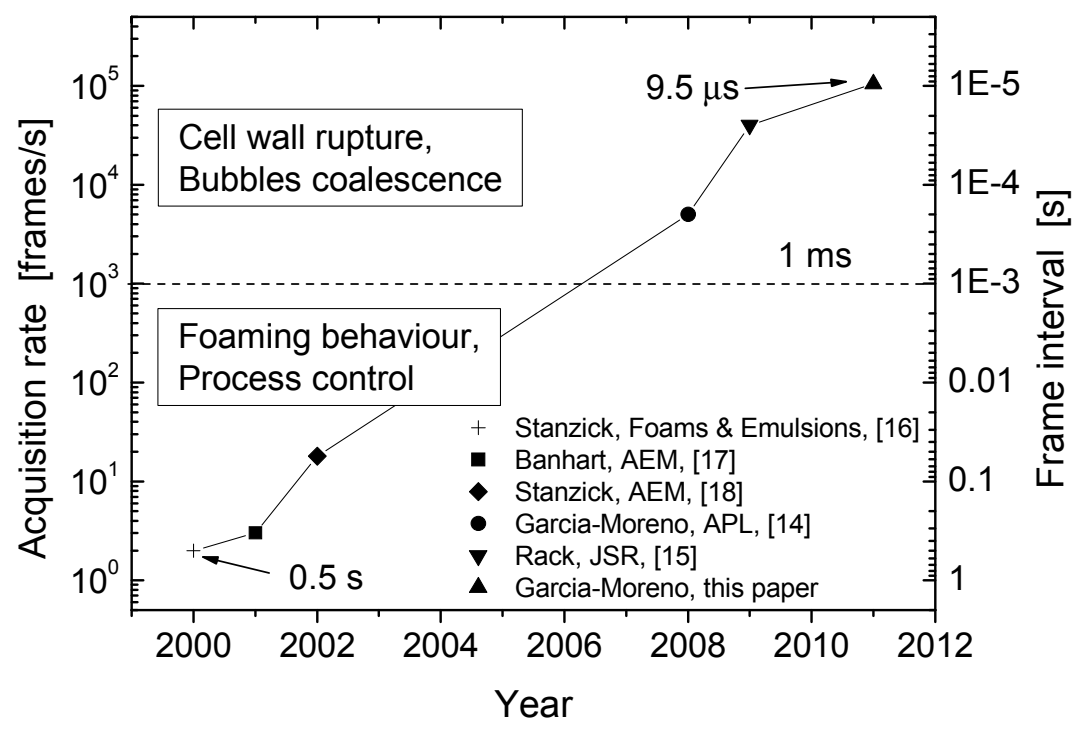




\section{Fast Synchrotron X-ray Radioscopy}

The stabilization of metal films - or cell walls - in liquid foams is a key issue in metal foam science but still not fully understood. More stable films imply less coalescence and therefore smaller bubbles with a more uniform size distribution, which is highly desirable. To investigate the nature of rupture, experiments have been performed in which there was a simultaneous demand for both high spatial and high time resolution. It was possible to observe the coalescence of two adjacent bubbles with 40,000 fps and an exposition time of $25 \mu$ s for each frame [15]. Recent experiments were conducted at a recording speed of $105,000 \mathrm{fps}$ with a frame interval of $9.5 \mu$ s and effective pixel size of $20 \mu \mathrm{m}$. Although the contrast of such images is low due to the short exposure time and consequent limited dynamics of the images, it is clearly visible in Figure 2 that the coalescence of two bubbles is completed in $\sim 475 \mu$ s and that the rupture of a film lasts for $\sim 380 \mu \mathrm{s}$, if we consider the end of the rupture as the point, where the contour of the new bubble becomes straight before it ends as convex.

Figure 2. Series of radiographs of an $\mathrm{AlSi} 10+0.5 \mathrm{wt} . \% \mathrm{TiH}_{2}$ foam at $640{ }^{\circ} \mathrm{C}$ extracted from an in-situ fast synchrotron X-ray radioscopic analysis (data acquired at beamline ID19 of the European Synchrotron Radiation Facility (ESRF)). The coalescence of two bubbles measured with $9.5 \mu$ s frame interval (105 kfps) can be observed. Dashed lines indicate the contours of the bubbles and arrows indicate the corresponding ruptured cell wall.
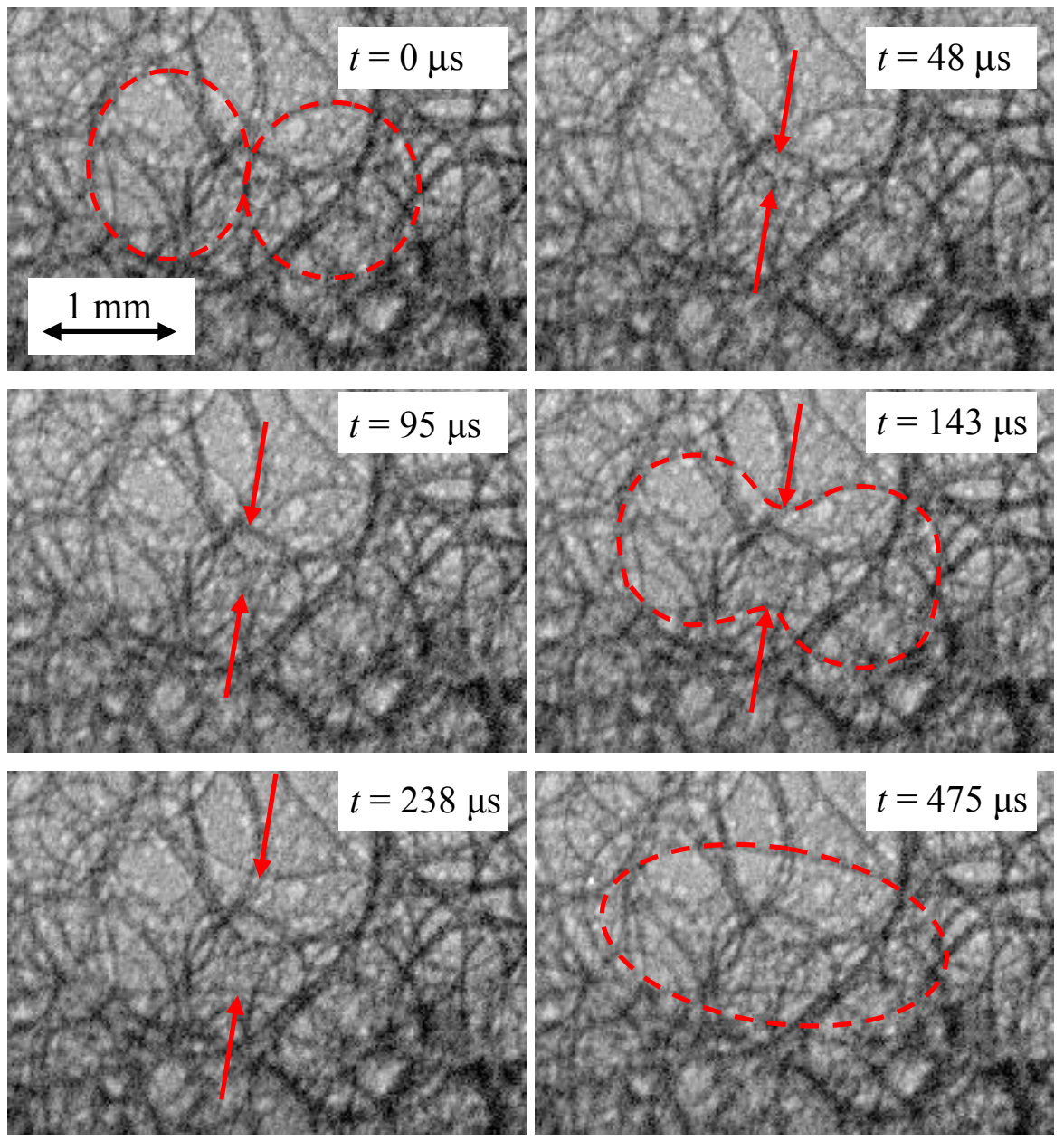
In this experiments it was possible to demonstrate that the rupture time of a film is dominated by the inertia of the fluid and not by its viscosity as rupture occurred so fast [14]. Therefore, it could be demonstrated that stabilization by an effective viscosity only, which would be as high as $\eta=0.4 \mathrm{~Pa} \cdot \mathrm{s}$ (as calculated by Gergely et al. [19]) does not apply to metal foams of the type investigated here. Other factors such as bubble size and alloy composition may influence the rupture time and will be studied in future.

\section{X-ray Laboratory Radioscopy-Selected Results}

In the year 2003, laboratory-based radioscopic equipment for in-situ studies of metal foam evolution went into operation at the Technical University Berlin jointly with the Helmholtz-Centre Berlin (formerly Hahn-Meitner Institute) [20]. Within the following years, this X-ray scanner was identified as a key instrument for the analysis of metal foaming. Various sample environments including fast heating plates and infrared furnaces, gas injection furnaces and pressure chambers were built. In addition, software was developed to extract as much quantitative information as possible quantitative information from image sequences, e.g., the course of foam expansion or density distributions. Furthermore, a cell wall rupture recognition module was developed that provides the temporal evolution and spatial distribution of coalescence [20,21]. In 2006, Brunke et al. described an experiment that was used to study metal foaming, especially foam evolution and drainage [22]. Their X-ray radioscopy setup was equipped with a cylindrical boron nitride $(\mathrm{BN})$ crucible that could be rotated to compensate gravitationally driven drainage. In 2007, a compact metal foaming furnace with very high temperature homogeneity $( \pm 1 \mathrm{~K})$ combined with X-ray diagnostics was constructed together with the European Space Agency and the Swedish Space Corporation for performing experiments under microgravity [23]. This setup has been used until now in two parabolic flight campaigns and one sounding rocket experiment [24]. Some of the results will be discussed in Section 4.5. The main advantage of such a laboratory-based equipment in comparison to synchrotron facilities is its availability and low cost. Some selected fields of application of the setup are summarized in this section and selected examples are presented.

\subsection{Process Control}

One of the advantages of using X-ray radioscopy is the possibility to observe qualitatively various effects of the foaming process in-situ even inside a foaming mold. This can provide a better process control. For example, one can observe the growth of nucleated bubbles, defect formations and evolution and the evolution of cracks or big pores [18]. Later, the influence of thermocouples acting as heat sinks on the heat transfer from the mold to the precursors, of the arrangement of the precursors, the mold filling process, density variations during process development, etc. were studied [25].

Figure 3 shows the inhomogeneous filling of a cylindrical mold at $700{ }^{\circ} \mathrm{C}$. This was caused by a temperature gradient of $\sim 100 \mathrm{~K}$ over $40 \mathrm{~mm}$ in the mold. The foam was generated from a rectangular bar of AlSi9 precursor placed in the center of the mold. As expected, the foam started foaming at the hottest part (left side) and continued expanding up to the right. This led to a broader, aged porous structure on the left and partially expanded foam on the right side. The resulting gradient in density and cell size can be directly observed in the X-ray images. 
Figure 3. X-ray radioscopic observation of the filling of a cylindrical steel mold by A1Si9 foam in an inhomogeneously heated precursor $\left(T=700^{\circ} \mathrm{C}, \Delta T \approx 2.5 \mathrm{~K} / \mathrm{mm}\right)$ at (a) $t=1 \mathrm{~s}$; (b) $t=110 \mathrm{~s} ;$ (c) $t=140 \mathrm{~s}$ and (d) $t=190 \mathrm{~s}$.

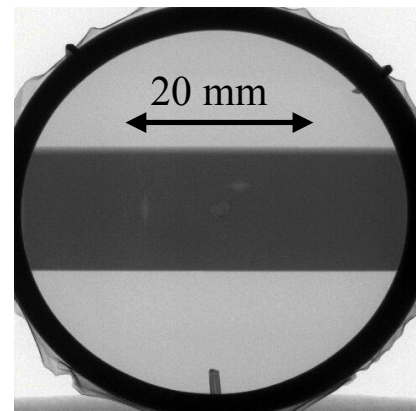

(a)

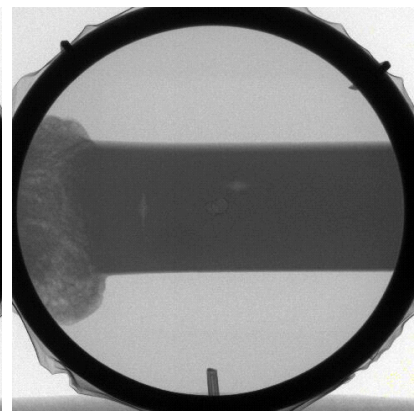

(b)

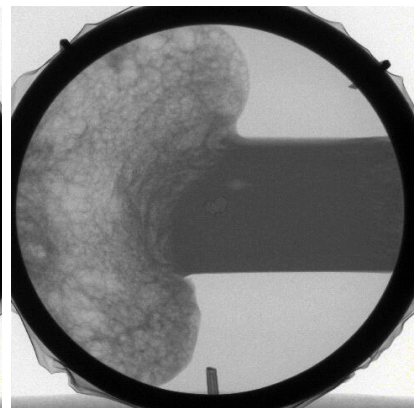

(c)

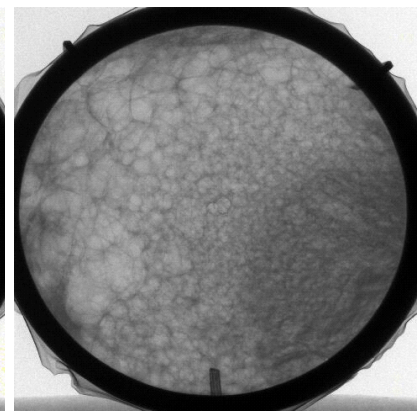

(d)

\subsection{New Effects}

Radioscopy has provided a deeper insight into foam evolution that allowed for the discovery of new effects. One of those was an unexpected behavior during solidification. In contrast to mechanical or laser expandometers, X-ray radioscopy, due to its high sensitivity, allowed us to find the new effect named "solidification expansion" [26]. It consists of an additional temporary expansion of the metal foam during solidification, where one would actually expect shrinkage as both the gas in the bubble and the metallic matrix shrink in the course of temperature reduction and solidification. The explanation of this counterintuitive phenomenon was found as a distortion of the balance between gas production and gas loses in the foam during solidification caused by the change in the gas-diffusion rate through the solidifying outer skin. This effect is usually small, e.g., a few percent of the total volume, but important because it takes place just during foam solidification and can induce, e.g., cell rupture.

Another interesting effect found was an alignment of the first line of bubbles next to the face sheets of aluminum foam sandwiches (AFS). By reducing the sandwich thickness to around three times the average bubble size, we could obtain an AFS with three aligned bubble layers (Figure 4). This alignment reduces the randomness of the pore distribution and makes foam properties more anisotropic.

Figure 4. X-ray radiography of an aluminum foam sandwich (AFS) produced by the company "metalfoam" exhibiting three vertically aligned bubble layers.

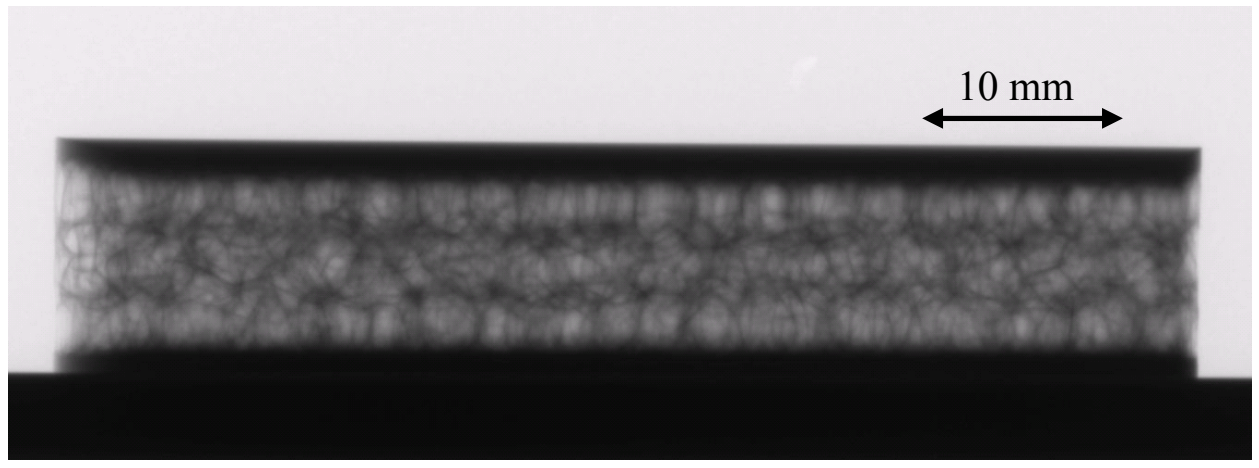




\subsection{Cell Wall Rupture}

To study the stability of foams in the liquid state, the coalescence rate calculated from the number of observed cell wall ruptures can be obtained quantitatively from X-ray image sequences. The dedicated software AXIM is able to compare subsequent images and to recognize individual ruptures [20]. The results are their spatial or temporal distributions, which provide information about, e.g., temperature dependence, drainage effects or long term stability [21,23,24].

\subsection{Foaming Under Controlled Atmosphere}

The influence of foaming atmosphere could be analyzed in-situ with X-ray radioscopy. It was found that in a non-oxidizing atmosphere the diffusion of hydrogen from the foam into the surrounding atmosphere is much faster, demonstrating that the collapse of the foam is mainly due to gas losses and not due to cell wall ruptures [27]. Figure 5 compares X-ray radiographies of two different Al samples at their maximum expansion and after cooling down to $400{ }^{\circ} \mathrm{C}$. They were foamed under air and argon atmosphere. The expansion plot (Figure 5e) shows that the foam produced in air is much more stable than the one produced under argon over the holding time of $600 \mathrm{~s}$, demonstrating the hydrogen retention capability of the oxide layer.

Figure 5. X-ray radiographies of two different $\mathrm{Al}+0.5$ wt. $\% \mathrm{TiH}_{2}$ foams produced (a); (b) in air and (c); (d) in argon at (a); (c) their maximum expansion and (b); (d) after cooling down to $400{ }^{\circ} \mathrm{C}$; (e) shows the temperature and area expansion courses, indicating the corresponding positions of the radiographies (a), (b), (c) and (d). The holding time is $600 \mathrm{~s}$.
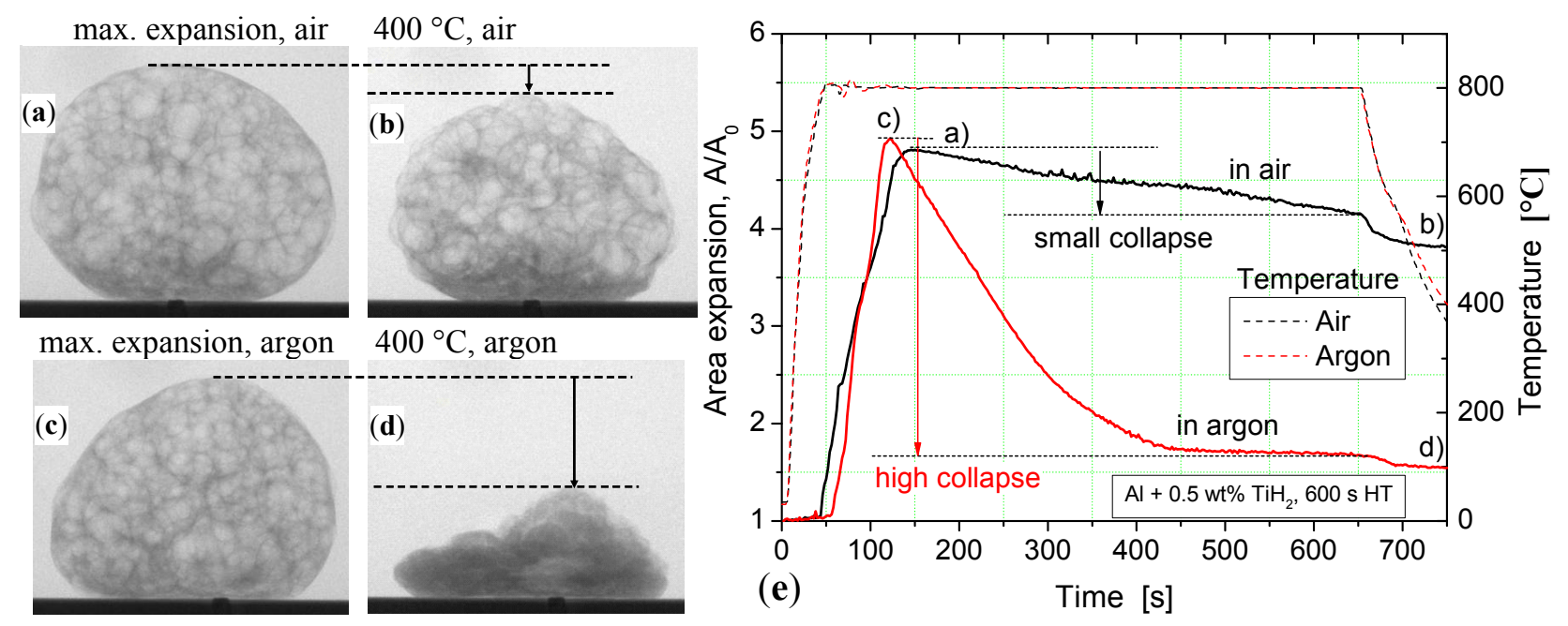

Not only the cover gas plays a role, but also the gas pressure. The effect of the surrounding pressure on foam nucleation and expansion was investigated [28-30]. An improved gas nucleation under high pressure was found. If the pressure is released in the liquid state, it leads to an increased expansion [30]. Variations of pressure showed a reversible behavior of liquid metal foams [29]. Radioscopic observations of the foaming of blowing agent-free powder compacts allowed us to demonstrate that the gas contained in the metal powders used for Al-based foams is sufficient for foaming if there are no gas losses during heating. This can be achieved by Pressure Induced Foaming 
(PIF), where powder compacts without any additional blowing agent are molten under high gas pressure (e.g., 5 bar) and foamed by subsequent pressure release to 1 bar [31].

Foaming of pure magnesium in argon as protective atmosphere was monitored in-situ with X-rays. This enabled us to find the conditions under which pure magnesium foams can be produced in a good quality following the standard PM-route. Mg-based foams are thought to have a potential for application in the field of biocompatible and biodegradable materials [32].

\subsection{Foaming Under Microgravity}

Foaming experiments under microgravity using inline X-ray diagnostics were performed for the first time in 2007 in the 46th parabolic flight campaign of the European Space Agency (ESA). The $\mathrm{X}$-ray radiographic images showed that fast liquid flow through a cellular structure is possible during strong gravity transitions [24]. Imbibition of drained liquid into a foam occurs under microgravity, leading to a homogeneously distributed liquid fraction. This demonstrates that capillary forces can compensate the effect of gravitational drainage. An even more challenging experiment was conducted during the MASER 11 sounding rocket campaign, where a liquid metal foam was analyzed during several minutes under microgravity (Figure 6). A comparison of the coalescence rate of the metal foamed under gravity and microgravity conditions showed that the total coalescence rate is almost the same in both conditions, although there is no drainage effect under microgravity [33]. This proved that gravity-driven drainage is not the main factor inducing cell wall instabilities, in contrast to what is known from aqueous foams.

Figure 6. X-ray radiographies of a thixocast AlSi6Cu4 +0.6 wt. $\% \mathrm{TiH}_{2}$ precursor foamed under (a) microgravity and (b) on Earth.
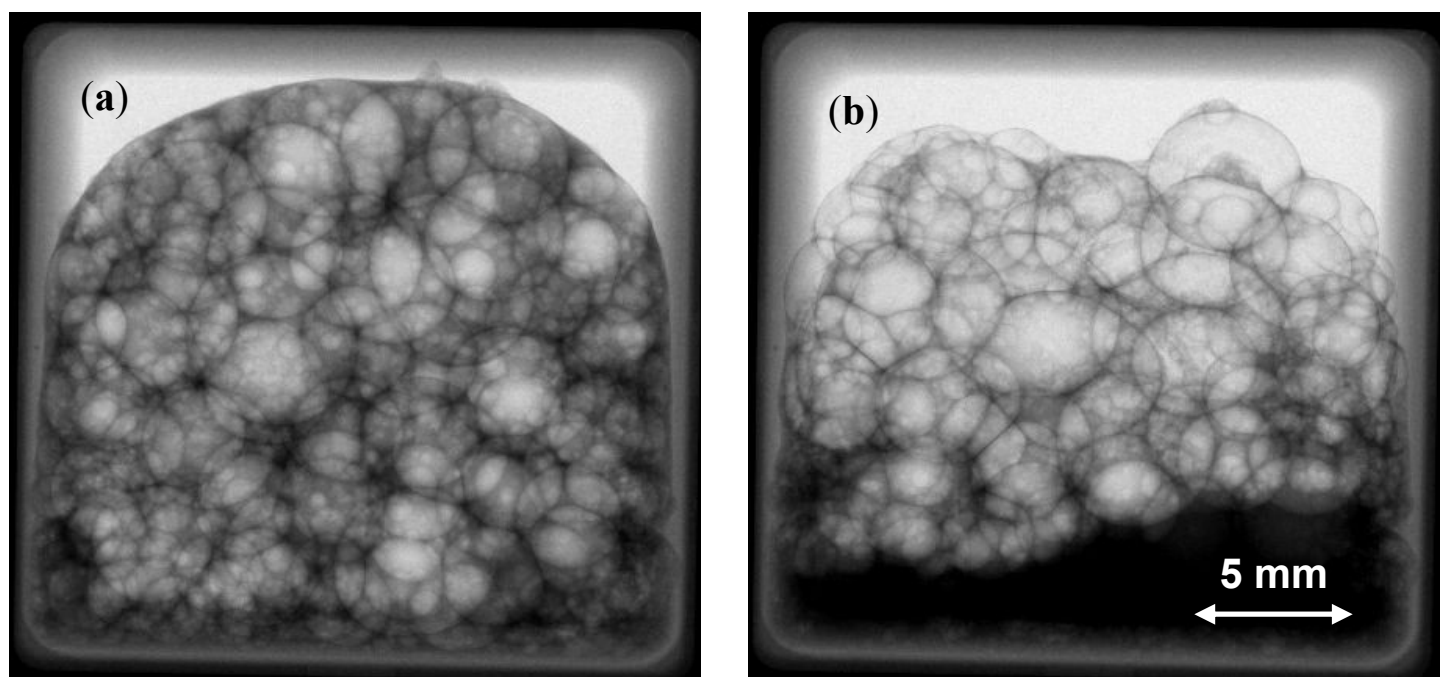

In 2009, in the frame of the 51st parabolic flight campaign of ESA, it could be confirmed that the blowing agent is the main destabilizing factor, thus shifting the emphasis of research to the analysis of the blowing agent kinetics and eventually to the further development of blowing agent free methods [31,34]. Figure 7a shows an X-ray radiography of a liquid $\mathrm{AlMg} 15 \mathrm{Cu} 10$ foam without extra blowing agent obtained in-situ during a microgravity experiment. In this alloy the intrinsic gas sources of the metal powders provide adequate amount of gas for foaming. The homogeneous gas nucleation 
and the reduced amount of cell wall ruptures lead to a very homogeneous pore structure, as can also be directly observed in the tomography (Figure 6b) or in a photograph of the polished surface structure (Figure 6c).

Figure 7. (a) In-situ X-ray radiography; (b) tomography and (c) photograph of a section of an $\mathrm{AlMg} 15 \mathrm{Cu} 10$ foam produced without adding a blowing agent.

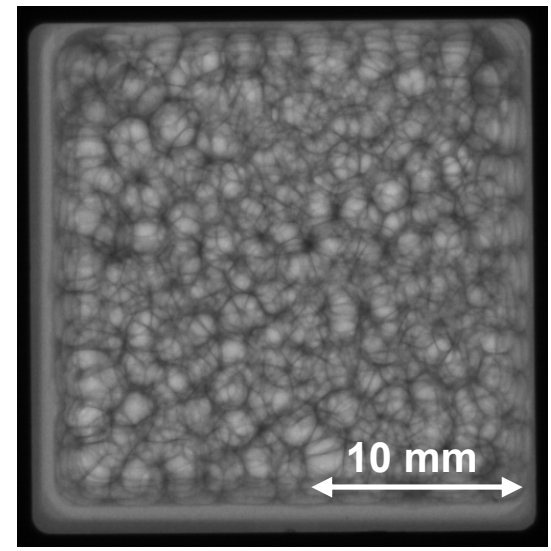

(a)

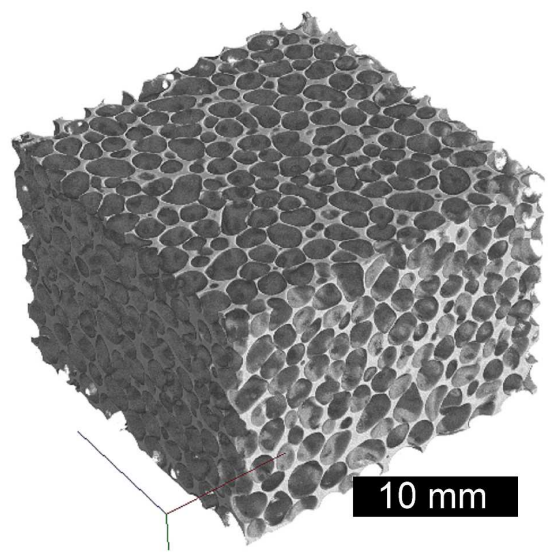

(b)

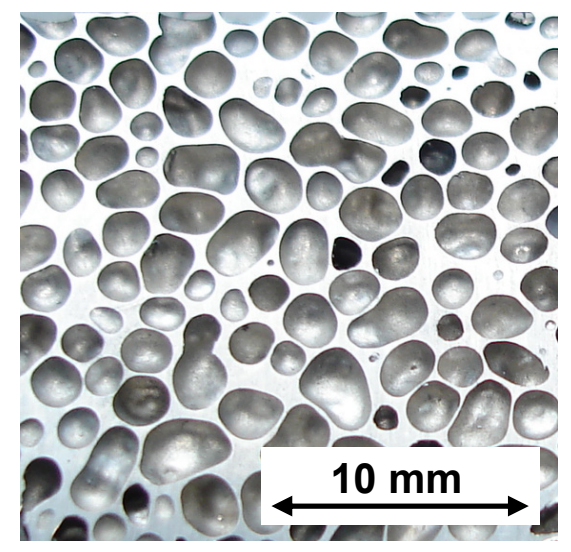

(c)

\section{Summary}

X-ray radioscopy is a powerful tool for the in-situ study of evolving metal foams. Both synchrotron and laboratory-based facilities allow for in-situ monitoring of foaming. High spatial resolution with an effective pixel size down to $20 \mu \mathrm{m}$ combined with the currently best achievable time resolution down to $10 \mu$ s allows us to detect and analyze cell wall ruptures and bubble coalescence. Foam expansion, mold filling, drainage, etc. could be monitored in-situ. The study of the influence of gas atmosphere and pressure allowed us to demonstrate the effect of oxidation on the gas loss through the outer foam skin. New foaming processes such as pressure-induced foaming or foaming with an intrinsic gas source were developed based on the results obtained from such experiments. Not only qualitative but also quantitative image analyses in terms of expansion evolution, density profiles, coalescence rate, drainage, etc. were performed. New effects such as solidification expansion and alignment of bubbles in aluminum foam sandwich panels were discovered. Experiments under microgravity used X-ray radioscopy to observe and quantify the imbibition of melt into foam under microgravity conditions and revealed the unexpectedly weak effect of gravity on coalescence.

\section{Acknowledgments}

Funding by the European Space Agency ESA (Project $\mu$ g-FOAM AO-99-075) is gratefully acknowledged as well as the European Synchrotron Radiation Facility (ESRF) for granting the beam time at ID19 (MA-1134). The experiments at ID19 were performed jointly with Lukas Helfen (ANKA). 


\section{References}

1. Banhart, J. Manufacture, characterization and application of cellular metals and metal foams. Prog. Mater. Sci. 2001, 46, 559-632.

2. Banhart, J.; Weaire, D. On the road again: Metal foams find favor. Phys. Today 2002, 55, 37-42.

3. Banhart, J.; Borbély, A.; Dzieciol, K.; García-Moreno, F.; Manke, I.; Kardjilov, N.; Kaysser-Pyzalla, A.R.; Strobl, M.; Treimer, W. X-ray and neutron imaging-Complementary techniques for materials science and engineering. Int. J. Mater. Res. 2010, 101, 1069-1079.

4. Mathiesen, R.H.; Arnberg, L.; Ramsøskar, K.; Weitkamp, T.; Raur, C.; Snigirev, A. Time-resolved X-ray imaging of Aluminum alloy solidification process. Metall. Mater. Trans. B 2002, 33, 613-623.

5. Buffet, A.; Reinhardt, G.; Schenk, T.; Nguyen-Thi, H.; Gastaldi, J.; Mangelinck-Noel, N.; Bergeon, N.; Jung, H.; Hartwig, J.; Baruchel, J. Real-time and in situ solidification of Al-based alloys investigated by synchrotron radiation: a unique experimental set-up combining radiography and topography techniques. Phys. Status Solidi A 2007, 204, 2721-2727.

6. Arnberg L.; Mathiesen, R.H. The real time, high-resolution X-ray video microscopy of solidification in aluminum alloys. JOM J. Miner. Met. Mater. Soc. 2007, 59, 20-26.

7. Nguyen-Thi, H.; Reinhardt, G.; Buffet, A.; Schenk, T.; Mangelinck-Noel, N.; Jung, H.; Bergeon, N.; Billia, B.; Hartwig J.; Baruchel, J. In situ and real time analysis of TGZM phenomena by synchrotron X-ray radiography. J. Cryst. Growth 2008, 310, 2906-2914.

8. Yasuda, H.; Yamamoto, Y.; Nakatsuka, N.; Yoshiya, M.; Nagira, T.; Sugiyama, A.; Ohnaka, I.; Uesugi, K.; Umetani, K. In situ observation of solidification in A-Cu and Fe-Si-Al alloys. Int. J. Cast. Met. Res. 2009, 22, 15-21.

9. Griesche, A.; Garcia-Moreno, F.; Macht, M.-P.; Frohberg, G. Chemical diffusion experiments in AlNiCe-melts. Mater. Sci. Forum 2006, 508, 567-572.

10. Zabler, S.; Rueda, A.; Rack, A.; Riesemeier, H.; Zaslansky, P.; Manke, I.; Garcia-Moreno, F.; Banhart, J. Coarsening of grain refined semi-solid Al-Ge32 alloy: X-ray micotomography and in-situ radiography. Acta Mater. 2007, 55, 5045-5055.

11. Zabler, S.; Rack, A.; Rueda, A.; Helfen, L.; Garcia-Moreno, F.; Banhart, J. Direct observation of flow in semi-solid alloys by synchrotron-based X-ray micro-radioscopy. Phys. Status Solidi A 2010, 207, 718-723.

12. Wang, Y.; Liu, X.; Im, K.S.; Lee, W.K.; Wang, J.; Fezzaa, K.; Hung, D.L.S.; Winkelman, J.R. Ultrafast X-ray study of dense-liquid-jet flow dynamics using structure-tracking velocimetry. Nat. Phys. 2008, 4, 305-309.

13. Rack, A.; Garcia-Moreno, F.; Schmitt, C.; Betz, O.; Cecilia, A.; Ershov, A.; Rack, T.; Banhart, J.; Zabler, S. On the possibilities of hard X-ray imaging with high spatio-temporal resolution using polychromatic synchrotron radiation. J. X-ray Sci. Technol. 2010, 18, 429-444.

14. Garcia-Moreno, F.; Rack, A.; Helfen, L.; Baumbach, T.; Zabler, S.; Babcsán, N.; Banhart, J.; Martin, T.; Ponchut, C.; di Michiel, M. Fast processes in liquid metal foams investigated by high-speed synchrotron X-ray micro-radioscopy. Appl. Phys. Lett. 2008, 92, 134104-134106. 
15. Rack, A.; Garcia-Moreno, F.; Baumbach, T.; Banhart, J. Synchrotron-based radioscopy employing spatio-temporal micro-resolution for studying fast phenomena in liquid metal foams. J. Synchrotron Radiat. 2009, 16, 432-434.

16. Stanzick, H.; Banhart, J.; Helfen, L.; Baumbach, T. In-situ monitoring of metal foam evolution and decay. In Foams and Emulsions; Zitha, P., Banhart, J., Verbist, G., Eds.; MIT-Verlag: Bremen, Germany, 2000; pp. 290-296.

17. Banhart, J.; Stanzick, H.; Helfen, L.; Baumbach, T.; Nijhof, K. Real-time X-ray investigation of Aluminium Foam Sandwich production. Adv. Eng. Mater. 2001, 3, 407-411.

18. Stanzick, H.; Wichmann, M.; Weise, J.; Helfen, L.; Baumbach, T.; Banhart, J. Process control in aluminium foam production using real-time X-ray radioscopy. Adv. Eng. Mater. 2002, 4, 814-823.

19. Gergely V.; Clyne, T.W. Drainage in standing liquid metal foams: Modelling and experimental observations. Acta Mater. 2004, 52, 3047-3058.

20. Garcia Moreno, F.; Fromme, M.; Banhart, J. Real-time X-ray radioscopy on metallic foams using a compact micro-focus source. Adv. Eng. Mater. 2004, 6, 416-420.

21. Garcia-Moreno, F.; Solórzano, E.; Banhart, J. Kinetics of coalescence in liquid aluminium foams. Soft Matter 2011, 7, 9216-9223.

22. Brunke O.; Odenbach, S. In situ observation and numerical calculations of the evolution of metallic foams. J. Phys. Condens. Matter 2006, 18, 6493-6506.

23. Garcia-Moreno, F.; Holm, P.; Banhart, J. Metallic foam experiments under microgravity. ESA Spec. Publ. 2007, 647, 389-392.

24. Garcia-Moreno, F.; Jiménez, C.; Mukherjee, M.; Holm, P.; Weise J.; Banhart, J. Experiments on metallic foams under gravity and microgravity. Colloids Surf. A 2009, 344, 101-106.

25. Garcia-Moreno, F.; Raffaele, N.; Banhart, J. Optimisation of mould filling during PM metal foaming. In Proceedings of the CellMet08; Stephani, G., Ed.; Dresden, Germany, 8-10 October 2008; pp. 33-138.

26. Mukherjee, M.; Garcia-Moreno, F.; Banhart, J. Solidification of metal foams. Acta Mater. 2010, $58,6358-6370$.

27. Mukherjee, M.; Garcia-Moreno, F.; Banhart, J. Collapse of aluminium foam in two different atmospheres. Metall. Mater. Trans. B 2010, 41, 500-504.

28. Körner, C.; Berger, F.; Arnold, M.; Stadelmann, C.; Singer, R.F. Influence of processing conditions on morphology of metal foams produced from metal powder. Mater. Sci. Technol. 2000, 16, 781-784.

29. Garcia-Moreno, F.; Babscán, N.; Banhart, J. X-ray radioscopy of liquid metal foams: Influence of heating profile, atmosphere and pressure. Colloids Surf. A 2005, 263, 290-294.

30. Garcia-Moreno, F.; Babcsán, N.; Banhart, J. The role of the gas pressure on the foaming of metals following the PM-route. In Porous Metals and Metal Foaming Technology; Nakajima, H., Kanetake, N., Eds.; The Japan Institute of Metals: Sendai, Japan, 2006; pp. 129-132.

31. Garcia-Moreno, F.; Banhart, J. Foaming of blowing agent-free aluminium powder compacts. Colloids Surf. A 2007, 309, 264-269.

32. Neu, T. Schäumen von Magnesium und Magnesiumlegierungen. Bachelor Thesis, Technical University Berlin, Berlin, Germany, 2011. 
33. Garcia-Moreno, F.; Mukherjee, M.; Jiménez, C.; Banhart, J. X-ray radioscopy of liquid metal foams under microgravity. Trans. Indian Inst. Met. 2009, 62, 451-454.

34. Mukherjee, M.; Garcia-Moreno, F.; Jiménez, C.; Banhart, J. Al and Zn foams blown by an intrinsic gas source. Adv. Eng. Mater. 2010, 12, 472-477.

(C) 2012 by the authors; licensee MDPI, Basel, Switzerland. This article is an open access article distributed under the terms and conditions of the Creative Commons Attribution license (http://creativecommons.org/licenses/by/3.0/). 